\title{
Diacronie
}

Studi di Storia Contemporanea

$N^{\circ} 31,3 \mid 2017$

"Le armi della politica, la politica delle armi"

\section{Alessandro Crocco, Il Risorgimento tra rivoluzioni e canzoni}

Michele Toss

\section{Q OpenEdition}

Journals

Edizione digitale

URL: http://journals.openedition.org/diacronie/6330

DOI: 10.4000/diacronie.6330

ISSN: 2038-0925

Editore

Association culturelle Diacronie

Notizia bibliografica digitale

Michele Toss, "Alessandro Crocco, II Risorgimento tra rivoluzioni e canzoni », Diacronie [Online], N31,

3 | 2017, documento 8, Messo online il 29 octobre 2017, consultato il 24 septembre 2020. URL : http:// journals.openedition.org/diacronie/6330; DOI : https://doi.org/10.4000/diacronie.6330 


\section{Diacronie}

Studi di Storia Contemporanea

$31,3 / 2017$

"Le armi della politica, la politica delle armi". Ideologie di lotta ed esperienze di guerra

\section{RECENSIONE: Alessandro CROCCO, Il Risorgimento tra rivoluzioni e canzoni, Civitavecchia, Prospettivaeditrice, 2016, 137 pp.}

\section{A cura di Michele TOSS}

Per citare questo articolo:

TOSS, Michele, «RECENSIONE: Alessandro CROCCO, Il Risorgimento tra rivoluzioni e canzoni, Civitavecchia, Prospettivaeditrice, 2016, 137 pp.», Diacronie. Studi di Storia Contemporanea : "Le armi della politica, la politica delle armi". Ideologie di lotta ed esperienze di guerra, 31, 3/2017, 29/10/2017,

URL: <http://www.studistorici.com/2017/10/29/toss_numero_31/>

Diacronie Studi di Storia Contemporanea $\rightarrow$ http://www.diacronie.it Rivista storica online. Uscita trimestrale.

redazione.diacronie@hotmail.it

Comitato di direzione: Naor Ben-Yehoyada - João Fábio Bertonha - Christopher Denis-Delacour - Maximiliano Fuentes Codera Anders Granås Kjøstvedt - John Paul Newman - Deborah Paci - Niccolò Pianciola - Spyridon Ploumidis - Wilko Graf Von Hardenberg

Comitato di redazione: Jacopo Bassi - Luca Bufarale - Gianluca Canè - Fausto Pietrancosta - Alessandro Salvador - Matteo Tomasoni Diritti: gli articoli di Diacronie. Studi di Storia Contemporanea sono pubblicati sotto licenza Creative Commons 3.0. Possono essere

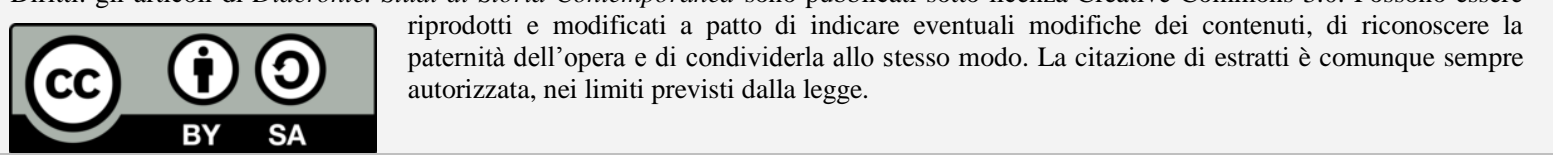




\title{
8/ RECENSIONE: Alessandro CROCCO, Il Risorgimento tra rivoluzioni e canzoni, Civitavecchia, Prospettivaeditrice, 2016, $137 \mathrm{pp}$.
}

\author{
A cura di Michele TOSS
}

«Del resto l'Ottocento è certamente il secolo di far nazioni. Ma è anche il secolo di far canzoni» ${ }^{1}$. Il volume di Alessandro Crocco è, a tal proposito, significativo. Professore di discipline letterarie e di latino nei licei, Crocco ha pubblicato saggi e articoli di storia contemporanea riguardanti in particolare la tradizione del canto politico e sociale nella cultura popolare italiana ${ }^{2}$. La storiografia degli ultimi anni ha già messo in luce l'importanza della canzone all'interno dell'analisi storica. Stefano Pivato, infatti, ha sottolineato come «il canto risulta essere un “documento" utile per capire la storia» ${ }^{3}$. Se poi si sposta lo sguardo al di fuori della disciplina prettamente storica, appaiono di maggior interesse e d'innovazione alcuni recenti lavori di cultural studies italiani che - riprendendo gli studi sulla popular music ${ }^{4}$ - hanno introdotto nuove importanti questioni e inediti approcci allo studio della canzone, come ad esempio la produzione musicale, i contesti di fruizione e di circolazione della musica e, più in generale, l'interpretazione del canto come una pratica sociale ${ }^{5}$.

All'interno dell'opera, la canzone viene analizzata da un punto di vista testuale e contenutistico e utilizzata come una vera e propria fonte per ricostruire le principali tappe del

\footnotetext{
${ }^{1}$ PIVATO, Stefano, «Inni e ballate», in CECCHINATO, Eva, ISNENGHI, Mario (a cura di), Fare l'Italia. Unità e disunità nel Risorgimento, vol. 1, Gli italiani in guerra. Conflitti, identità, memorie, Torino, Utet, 2008, pp. 660-673, p. 663.

${ }^{2}$ Si possono citare, oltre al presente volume: CROCCO, Alessandro, Maramao perché sei morto: la seconda guerra mondiale nelle canzoni, Bari, Palomar, 2010; ID., "L'immagine di Garibaldi nei canti del Risorgimento», in Chronica mundi, 2, 2/2011, pp. 22-41.

${ }^{3}$ PIVATO, Stefano, Bella ciao. Canto e politica nella storia d'Italia, Roma-Bari, Laterza, 2005, p. X. Per l'utilizzazione del canto all'interno di studi politico-sociali al di fuori dei confini nazionali si rinvia ad esempio a KÖRNER, Axel, Das Lied von einer anderen Welt. Kulturelle Praxis im französichen und deutschen Arbeitermilieu 1840-1890, Frankfurt am Main, Campus Verlag, 1997; DARRIULAT, Philippe, La muse du peuple. Chansons politiques et sociales en France 1815-1871, Rennes, Presses Universitaires de Rennes, 2010; GANEV, Robin, Songs of Protest, Song of Love. Popular Ballads in Eighteenth-Century Britain, Manchester, Manchester University Press, 2009.

${ }^{4}$ MIDDLETON, Richard, Studiare la popular music, Milano, Feltrinelli, 1994.

${ }^{5}$ FABBRI, Franco, Around the clock. Una breve storia della popular music, Torino, Utet, 2008; ID., Il suono in cui viviamo. Saggi sulla popular music, Milano, Il Saggiatore, 2008; FANELLI, Antonio, Contro canto. Le culture della protesta dal canto sociale al rap, Roma, Donzelli, 2017.
} 
Risorgimento italiano. Il libro segue un filo cronologico che conduce il lettore dagli esordi del movimento nazionale del periodo giacobino fino alla soglia degli anni Settanta dell'Ottocento, passando per i rivolgimenti del '48 e le guerre d'Indipendenza. Si tratta di un lavoro di sintesi, tratto dalla tesi di laurea dell'autore ${ }^{6}$, che si traduce in una lunga carrellata di canti che Crocco annota e contestualizza ${ }^{7}$.

Le fonti utilizzate sono ampie e diversificate tra loro: i canti di produzione colta, composti cioè $\mathrm{da}$ autori di professione che spesso utilizzavano espressioni auliche e retoriche, quelli popolareggianti, che rielaboravano le parole d'ordine patriottiche mescolando il linguaggio "alto" e "basso", e i testi più popolari, spesso anonimi, formati da una grande varietà di canti minori, strofette e canzonette (molte delle quali in dialetto). Uno dei meriti dell'autore è di aver preso in considerazione anche i canti delle regioni meridionali, proponendoli nella loro versione originale e in traduzione ${ }^{8}$; spesso, infatti, gli studi sul canto politico e sociale hanno privilegiato l'area geografica centro-settentrionale.

Spostando l'attenzione sul taglio interpretativo proposto nell'opera, di particolare interesse è la parte dedicata alla rappresentazione di Giuseppe Garibaldi all'interno del canto risorgimentale 9 Una personalità complessa che fu al centro di istanze differenti tra loro: «in lui» - scrive l'autore «erano riposte le speranze sia di chi auspicava la creazione di una repubblica in Italia sia di chi lo vedeva come il coraggioso combattente al servizio della monarchia sabauda sia di chi infine lo considerava un giustiziere popolare ${ }^{10}$. Come ha sottolineato Maurizio Degl'Innocenti, Garibaldi «dava corpo a tante attese, anche di diverso orientamento» ${ }^{11}$. L'analisi di Crocco mette in luce gli aggettivi e le immagini utilizzate nei canti di questo periodo per definire l'eroe dei due mondi. «Qual è il guerriero famoso al pari / Di qua d'Atlante, di là dai mari, / che per l'Italia brandì l'acciaro, / e il nostro nome fè sacro e caro / fin fra' selvaggi nudi e spavaldi ? / È Garibaldi» ${ }^{12}$ : così recitava una strofa del canto intitolato Garibaldi composto da Francesco Dall'Ongaro. «Guerriero», «liberatore», «salvatore» sono solo alcuni degli epiteti utilizzati per riferirsi al nizzardo ${ }^{13}$. La fama

\footnotetext{
${ }^{6}$ CROCCO, Alessandro, "Patrioti all'Alpe andiamo". Il Risorgimento tra rivoluzioni e canzoni, tesi di laurea specialistica in Filologia moderna, Università di Catania, a.a. 2009-2010.

${ }^{7}$ Le note al testo sono molte limitate e non contengono i necessari riferimenti bibliografici per risalire alle opere dalle quali sono stati presi i numerosi canti proposti. Il volume, tuttavia, è corredato da un utile indice delle canzoni citate suddiviso per titolo o per incipit che favorisce la ricerca.

${ }^{8} \mathrm{Nel}$ testo, ad esempio, vengono ampiamente utilizzate le raccolte di canti siciliani di studiosi come Leonardo Vigo, Giuseppe Pitrè, Salvatore Salomone Marino e Antonino Uccello.

${ }^{9}$ Una tematica già affrontata dall'autore in CROCCO, Alessandro, «L'immagine di Garibaldi nei canti del risorgimento", cit.

${ }^{10}$ CROCCO, Alessandro, Il Risorgimento tra rivoluzioni e canzoni, cit., p. 68.

${ }^{11}$ DEGL'INNOCENTI, Maurizio, Garibaldi e l'Ottocento. Nazione, popolo, volontariato, associazione, Manduria-BariRoma, Lacaita, 2008, pp. 226.

${ }^{12}$ CROCCO, Alessandro, Il Risorgimento tra rivoluzioni e canzoni, cit., p. 73.

${ }^{13}$ A tal proposito mi permetto di rinviare ad alcune riflessioni di chi scrive, cfr. TOSS, Michele, "Quando la tromba suonava all'armi / Con Garibaldi corsi a arruolarmi". L'immagine di Garibaldi nel canto popolare di epoca risorgimentale», in CASALENA, Maria Pia (a cura di), Luoghi d'Europa: Culti, città, economie, Bologna,
} 
di Garibaldi fu talmente ampia e radicata nella popolazione da trasformarlo in un'icona popolare, in un eroe, in un vero e proprio mito e in un oggetto di culto ${ }^{14}$. Prevalentemente in ambito meridionale, ma non solo, molti canti si riferivano a Garibaldi utilizzando delle immagini e un linguaggio legati alla sfera religiosa. Egli assumeva quindi i tratti di un messia dalle capacità sovrumane e con poteri magici, un santo con caratteristiche quasi divine: «Ch'è beddu Caribardi che mi pari / san Micheluzzo arcancilu davero, / la Sicilia la vinni a libbirari / e vinnicari a chiddi ca mureru, / quannu talìa Gesù Cristu mi pari, / quannu cumanna Carlu Magnu veru» ${ }^{15}$.

La centralità della figura di Garibaldi derivava non solo dagli apprezzamenti messi in rima dai suoi estimatori; egli divenne il protagonista anche delle invettive cantate dagli avversari. Al termine del processo risorgimentale, soprattutto nelle regioni meridionali, iniziò a serpeggiare l'accusa di traditore rivolta a Garibaldi. L'unificazione italiana non aveva mantenuto le promesse di un miglioramento delle condizioni di vita e «una parte del rancore popolare fu diretto inevitabilmente contro Garibaldi che, ai loro occhi, li aveva illusi: Ca amm'a fa de Garebbalde / ca iè mbane e tradètore ? / Ne valìme u rè Berbòne / ca respètte la religgione» ${ }^{16}$.

L'autore inserisce queste tipologie di strofe all'interno di un contesto più ampio, quello dei canti legati alla tradizione antigiacobina, sanfedista e antirisorgimentale. L'ultimo capitolo dell'opera di Crocco, infatti, è dedicato proprio alle canzoni reazionarie e di malcontento che si diffusero tra la popolazione a partire dal periodo napoleonico fino ai primi anni postunitari ${ }^{17}$. Rientrano all'interno di questa sezione i canti di chi si oppose al movimento risorgimentale in nome dei simboli e dei valori promossi dal clero e dalla chiesa. Sono molti gli esempi riportati nell'opera, come la canzone antigiacobina cantata dall'esercito controrivoluzionario del cardinale Ruffo contro la Repubblica di Napoli del 1799 A lu suono della gran cascia ${ }^{18}$, quelle diffuse in Toscana nel 1796 di Viva Maria, viva Gesù contro l'occupazione francese e in Piemonte, sempre negli stessi

Clueb, 2012, pp. 53-71.

${ }^{14}$ Sul mito di Garibaldi si rinvia a ISNENGHI, Mario, Garibaldi fu ferito: il mito, le favole, Roma, Donzelli, 2010; RIALL, Lucy, Garibaldi: the first modern celebrity, in RAGUSA, Andrea (a cura di), Giuseppe Garibaldi: un eroe popolare nell'Europa dell'Ottocento, Manduria, Lacaita, 2009, pp. 7-24; ID., Garibaldi. L'invenzione di un eroe, Roma-Bari, Laterza, 2007; DEGL'INNOCENTI, Maurizio, Garibaldi e l'Ottocento, cit.

${ }^{15}$ «Com'è bello Garibaldi che mi pare / San Micheluzzo arcangelo in persona, / la Sicilia è venuto a liberare / a vendicare quelli che sono morti, / quando guarda sembra Gesù Cristo, / quando comanda un vero Carlo Magno». CROCCO, Alessandro, Il Risorgimento tra rivoluzioni e canzoni, cit., p. 82.

${ }^{16}$ «Che cosa dobbiamo farne di Garibaldi / che è infame e traditore ? / Noi vogliamo il re Borbone / che rispetta la religione». Ibidem, p. 114.

17 A questo proposito i capitoli curati da Roberto Leydi «Sempre morte a' giacobini. Canti antigiacobini, antifrancese, sanfedisti e anti-risorgimentali», "Canti di delusione, malcontento e protesta nel primo periodo postunitario», «Partire partirò, partir bisogna. Canti militari contro la coscrizione e canti contro la guerra, dall'età napoleonica al primo periodo postunitario» costituiscono ancora una delle raccolte più esaustive su questi temi. Cfr. LEYDI, Roberto, Canti sociali italiani, Milano, Edizioni Avanti!, 1963, pp. 208-445 ${ }^{18}$ "A lu suono de le campane / Viva, viva li populane, / a lu suono de li viulini / sempre a morti à giacobini» trad. «Al suono delle campane / viva, viva i popolani / al suono dei violini / sempre a morte ai giacobini». CROCCO, Alessandro, Il Risorgimento tra rivoluzioni e canzoni, cit., p. 92. 
anni, come il canto Sti giacobin s'fazio razun ${ }^{19}$. Anche durante il periodo delle guerre d'indipendenza circolarono nella penisola canti che contenevano un forte carattere protestatario. Ne sono una testimonianza le strofette di «Viva Radeschi e viva Metternich, / morte ai sciuri e viva ai puverett ${ }^{20}$, cantate in seguito alle Cinque giornate di Milano, che dimostrano come una parte della popolazione (soprattutto contadina) identificò il movimento patriottico con la classe dei ricchi e dei signori. Più interessanti ancora sono quest'altre rime livornese di autore anonimo, composte contro il governo provvisorio toscano retto dai tre triumviri Montanelli, Guerrazzi e Mazzoni, che costituiscono uno schiaffo non solo alle istanze risorgimentali italiane e agli ideali di libertà, propagandati dai giacobini e dai francesi prima e successivamente dai liberali e dai piemontesi, ma anche a tutto quel movimento internazionale di emancipazione popolare in atto a partire dal '48 francese ${ }^{21}$ : «Diceva un codino / e aveva ragione, / che il re più minchione / è il Popolo-re. [...] / La misera storia / del danno sofferto, / ti renda più esperto, / più degno di te, / o re de' minchioni, / o Popolo-re»" ${ }^{22}$.

Non solo le strofette popolari, l'autore presenta anche i canti di tradizione colta e semicolta che possedevano una certa circolazione tra la popolazione. Per i canti popolareggianti diffusissimo tra i volontari italiani nel 1848-1849, ad esempio, era il canto di Luigi Mercantini Patrioti all'Alpe andiamo, il cui ritornello spronava e rinvigoriva gli animi dei combattenti durante le lunghe marce: «Foco, foco, foco, foco! / S'ha da vincere o morir. / Foco, foco, foco, foco! / Ma il tedesco ha da morir / E sol verde, bianca e rossa, / la bandiera s'innalzò. / E sol verde, bianca e rossa, la bandiera s'innalzò» ${ }^{23}$. La bandiera tricolore in quegli anni, infatti, stava diventando un vero e proprio simbolo di libertà: «La bandiera dei tre colori / è sempre stata la più bella, / noi vogliamo sempre quella, / noi vogliamo la libertà» ${ }^{24}$.

L'unificazione nazionale non pose certo termine ai contrasti interni al paese; molte furono le voci di insoddisfazione di fronte alla disillusione delle speranze di un reale cambiamento sociale. Crocco utilizza il canto per ottenere un accesso privilegiato a questi sentimenti di sfiducia. Tali componimenti esprimevano un malessere causato da un sistema politico che si percepiva distante dai bisogni della popolazione e che non corrispondeva alle attese che avevano animato molti patrioti: «Non capísciu cosa è stu Parramento, / siddu è 'ndiavulato o puru santu, / ca pàrtiri ni fa

\footnotetext{
${ }^{19}$ «Sti giacobin s'fazio razun, / vuréivo lvé la religiun. / Lur i fazio na gran festa, / a préive e frà cupé-i la testa. / La liberté l'è andà a la fin / A confüziun dëi giacobin [...] / O giacobin, l'éi vü na ruta, / E l'éi pià-ve na bela bota, / e giacobin e patriot / e vi bütruma tüti al crot» trad. "Questi giacobini si facevano ragione, / volevano levare la religione. / Facevano una gran festa / ai preti e ai frati tagliare la testa. / La libertà è andata alla fine / a confusione dei giacobini [...] / O giacobin, siete stati sconfitti, / vi pigliaste una bella botta / e giacobin e patrioti / vi butterem tutti in prigione». CROCCO Alessandro, Il Risorgimento tra rivoluzioni e canzoni, cit., p. 96.

${ }^{20}$ Ibidem, p. 98.

${ }^{21}$ Cfr. TOSS, Michele, Il Popolo Re. La canzone sociale a Parigi (1830-1848), Bologna, Clueb, 2013, pp. 153 et seq.

${ }^{22}$ CROCCO, Alessandro, Il Risorgimento tra rivoluzioni e canzoni, cit., p. 101.

${ }^{23}$ Ibidem, p. 42.

${ }^{24}$ Ibidem, p. 43.
} 
lu sentimentu: / misi sti pisi a fa paari tantu? / Centu stamu paanu e n'autri centu, / semu cu pigghia e paga tantu e tantu; / si buatri pigghiati, e nui pagumu, / no resta oru, argentu e no ramu» ${ }^{25}$.

${ }^{25}$ Vito Mangano, «Non capisciu cosa è stu Parramentu». Ibidem, p. 112. Trad. «Non capisco cosa è questo Parlamento, / se è un indemoniato oppure un santo; / ci fai smarrire il ben dell'intelletto: / c'impose pesi e fa pagare tanto? / Cento stiamo pagando e ancora cento, / qui c'è chi prende e chi paga tanto e tanto: / se voialtri prendete, e noi paghiamo, / non resto oro, né argento, né rame». 


\section{L'AUTORE}

Michele TOSS, laureato in storia contemporanea all'Ecole Pratique des Hautes Etudes, si è diplomato in storia all'Ecole Normale Supérieure di Parigi e successivamente ha ottenuto un dottorato in cotutela presso il dipartimento di Storia Culture Civiltà dell'Università di Bologna con una ricerca sul canto sociale in Italia e Francia nel XIX secolo. Attualmente collabora con la Fondazione Museo storico del Trentino con un progetto postdoc sulle pratiche d'opposizione popolare al fascismo in Trentino.

URL: < http://www.studistorici.com/progett/autori/\#Toss > 